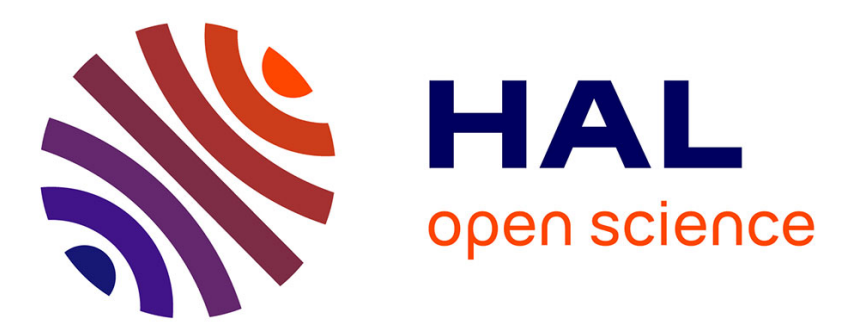

\title{
RELATION ENTRE L'APPROXIMATION DE SUPERPOSITION DE KIRKWOOD ET LES ÉQUATIONS HNC OU MSA POUR LES SOLUTIONS IONIQUES
}

\author{
Jean Pierre Badiali, J. Lestrade
}

\section{To cite this version:}

Jean Pierre Badiali, J. Lestrade. RELATION ENTRE L'APPROXIMATION DE SUPERPOSITION DE KIRKWOOD ET LES ÉQUATIONS HNC OU MSA POUR LES SOLUTIONS IONIQUES. Journal de Physique Colloques, 1978, 39 (C1), pp.191-195. 10.1051/jphyscol:1978137 . jpa-00217334

HAL Id: jpa-00217334

https://hal.science/jpa-00217334

Submitted on 1 Jan 1978

HAL is a multi-disciplinary open access archive for the deposit and dissemination of scientific research documents, whether they are published or not. The documents may come from teaching and research institutions in France or abroad, or from public or private research centers.
L'archive ouverte pluridisciplinaire HAL, est destinée au dépôt et à la diffusion de documents scientifiques de niveau recherche, publiés ou non, émanant des établissements d'enseignement et de recherche français ou étrangers, des laboratoires publics ou privés. 


\title{
RELATION ENTRE L'APPROXIMATION DE SUPERPOSITION DE KIRKWOOD ET LES ÉQUATIONS HNC OU MSA POUR LES SOLUTIONS IONIQUES
}

\author{
J. P. BADIALI et J. C. LESTRADE
}

Groupe de Recherche no 4 du C. N. R. S. «Physique des Liquides et Electrochimie » associé à l'Université P. et M. Curie, 4, place Jussieu, 75230 Paris Cedex 05, France

Résumé. - On présente une synthèse de différentes approches qui permettent de retrouver les équations HNC ou MSA sans utilisation de diagramme mais par itération d'une équation intégrale basée sur l'approximation de Kirkwood.

Abstract. - The authors give various approaches which allow HNC equations and the mean spherical approximation to be found without using diagrams. An integral equation based on the Kirkwood approximation is solved by iteration.

Parmi les systèmes physiques présentant des interactions coulombiennes, les solutions électrolytiques sont les premières qui ont donné lieu à une approche théorique. Dès 1923 Debye et Hückel [1] proposèrent une expression mathématique pour les fonctions de distribution radiale interionique $g_{\alpha \beta}(r)$. Depuis une vingtaine d'années un effort particulier a été effectué pour formuler la théorie des solutions ioniques dans le cadre du formalisme des développements en diagramme. Dans une publication récente Rasaiah [2] a fait le point sur ce type d'approche et comparé les prévisions théoriques aux données issues de calculs de Monte Carlo, que l'on convient de considérer comme des données de référence.

Les approches diagrammatiques ont supplanté les méthodes basées sur des solutions approchées d'équations intégrales exactes. Ces dernières méthodes reposent sur l'utilisation d'hypothèses physiques. Elles ont une base beaucoup plus phénoménologique; toutefois elles sont en général beaucoup plus accessibles au physico-chimiste. Dans un article de synthèse sur les propriétés des solutions diluées $\mathbf{H}$. Falkenhagen et W. Ebeling [3] ont reproduit quelques résultats obtenus dans cette direction. Il semble intéressant aujourd'hui de rappeler un certain nombre de liens existant entre les deux méthodes de calcul des fonctions de distribution radiale.

Dans une première partie nous rappellerons les définitions et les propriétés des différentes fonctions caractérisant la structure des solutions électrolytiques.
Ensuite, en utilisant systématiquement l'approximation de superposition de Kirkwood [4] nous montrerons que l'on peut en déduire simplement l'approximation sphérique moyenne (MSA) introduite pour les solutions ioniques par Waizman et Lebowitz [5]. On montrera dans la troisième partie de ce travail que l'approximation HNC (hypernetted chain), qui s'est révélée satisfaisante pour des solutions diluées et/ou relativement concentrées peut aussi être retrouvée simplement. Nous nous limitons ici au cas où le solvant est assimilé à un continuum diélectrique de permittivité $\varepsilon$. C'est ce que l'on appelle communément le modèle primitif des solutions électrolytiques.

1. Fonctions caractérisant la structure des solutions ioniques. - Pour caractériser la structure des liquides ioniques on peut utiliser indifféremment les fonctions de distribution radiale $g_{\alpha \beta}(r)$, les fonctions de corrélation

$$
h_{\alpha \beta}(r)=g_{\alpha \beta}(r)-1
$$

ou encore les fonctions de corrélation directe $C_{\alpha \beta}(r)$ définies par l'équation d'Ornstein Zernicke [5] :

$\dot{h}_{\alpha \beta}\left(r_{12}\right)=C_{\alpha \beta}\left(r_{12}\right)+\sum_{\gamma} n_{\gamma} \int h_{\alpha \gamma}\left(r_{13}\right) C_{\gamma \beta}\left(r_{32}\right) \mathrm{d} \mathbf{r}_{3}$

où $n_{\gamma}$ est la concentration de l'espèce $\gamma$. Par itération on peut exprimer $C_{\alpha \beta}\left(r_{12}\right)$ à partir des différentes fonctions de corrélation. On a en effet :

$$
\begin{aligned}
C_{\alpha \beta}\left(r_{12}\right)=h_{\alpha \beta}\left(r_{12}\right) & -\sum_{\gamma} n_{\gamma} \int h_{\alpha \gamma}\left(r_{13}\right) h_{\gamma \beta}\left(r_{32}\right) \mathrm{d} \mathbf{r}_{3} \\
& +\sum_{\gamma} \sum_{\delta} n_{\gamma} n_{\delta} \int h_{\alpha \gamma}\left(r_{13}\right) h_{\gamma \delta}\left(r_{34}\right) h_{\delta \beta}\left(r_{42}\right) \mathrm{d} \mathbf{r}_{3} \mathrm{~d} \mathbf{r}_{4}-\cdots
\end{aligned}
$$


Par intégration de l'équation de Liouville, on montre classiquement que $g_{\alpha \beta}\left(r_{12}\right)$ doit vérifier l'équation [6]:

$$
\begin{aligned}
k T \operatorname{grad}_{1} g_{\alpha \beta}\left(r_{12}\right) & +g_{\alpha \beta}\left(r_{12}\right) \cdot \operatorname{grad}_{1} V_{\alpha \beta}\left(r_{12}\right) \\
& +\sum_{\gamma} n_{\gamma} \int \operatorname{grad}_{1} V_{\alpha \gamma}\left(r_{13}\right) g_{\alpha \beta \gamma}\left(\mathbf{r}_{1} \mathbf{r}_{2} \mathbf{r}_{3}\right) \mathrm{d} \mathbf{r}_{3}=0
\end{aligned}
$$

où $V_{\alpha \beta}\left(r_{12}\right)$ est le potentiel d'interaction des ions $\alpha$ et $\beta$.

Cette équation établit une relation entre $g_{\alpha \beta}\left(r_{12}\right)$ et la fonction de corrélation à trois corps $g_{\alpha \beta \gamma}\left(\mathbf{r}_{1} \mathbf{r}_{2} \mathbf{r}_{3}\right)$. Une deuxième relation de ce type existe, elle résulte de la définition même de $g_{\alpha \beta \gamma}\left(\mathbf{r}_{1} \mathbf{r}_{2} \mathbf{r}_{3}\right)$, on a en effet [4] :

$$
\int g_{\alpha \beta \gamma}\left(\mathbf{r}_{1} \mathbf{r}_{2} \mathbf{r}_{3}\right) \mathrm{d} \mathbf{r}_{3}=V \cdot g_{\alpha \beta}\left(r_{12}\right)
$$

où $V$ est le volume de l'échantillon. Par ailleurs, la mécanique statistique montre que l'on doit avoir, lorsque la concentration de toutes les espèces d'ions tend vers zéro :

$$
g_{\alpha \beta}\left(r_{12}\right)=\exp -\beta V_{\alpha \beta}\left(r_{12}\right) \quad\left(\beta=\frac{1}{k T}\right) .
$$

Dans le cas des relations ioniques les différentes fonctions de distribution radiale ne peuvent être construites indépendamment les unes des autres. Il existe en effet deux relations entre ces grandeurs. La première assure, à l'équilibre, la neutralité électrique du milieu

$$
\sum_{\beta} \int n_{\beta} c_{\beta} g_{\alpha \beta}\left(r_{12}\right) \mathrm{d} \mathbf{r}_{12}=-c_{\alpha}
$$

où $c_{\alpha}$ est la charge de la particule $\alpha$. La seconde relation a été découverte par Stillinger et Lovett [7] et elle caractérise les milieux conducteurs :

$$
-6 \sum_{\alpha} C_{\alpha} c_{\alpha}^{2}=K_{\mathrm{D}}^{2} \int \sum_{\alpha} \sum_{\beta} n_{\alpha} n_{\beta} c_{\alpha} c_{\beta} r_{12}^{2} g_{\alpha \beta}\left(r_{12}\right) \mathrm{d} \mathbf{r}_{12} .
$$

Les équations (4)-(8) constituent des contraintes que les modèles de fonction de distribution radiale doivent respecter. En général, pour un modèle donné, ces équations ne sont pas toutes vérifiées. Par exemple, l'approximation de superposition de Kirkwood [4]

$$
g_{\alpha \beta \gamma}\left(\mathbf{r}_{1} \mathbf{r}_{2} \mathbf{r}_{3}\right)=g_{\alpha \beta}\left(r_{12}\right) g_{\beta \gamma}\left(r_{23}\right) g_{\gamma \alpha}\left(r_{31}\right)
$$

portée dans (5) conduit à

$$
\int g_{\beta \gamma}\left(r_{23}\right) g_{\gamma \alpha}\left(r_{31}\right) \mathrm{d} \mathbf{r}_{3}=V .
$$

Une telle relation n'est manifestement vérifiée que si $g_{\beta \gamma}\left(r_{23}\right)=1$ et $g_{\gamma \alpha}\left(r_{31}\right)=1$. Ceci montre que l'approximation (9) ne peut être satisfaisante que dans le cas limite où les différentes concentrations $n_{y}$ tendent vers zéro [4]. On peut alors considérer que les distances interparticulaires sont en moyenne, telles que :

$$
\begin{aligned}
& r_{13} \rightarrow \infty \\
& r_{12} \rightarrow \infty \\
& r_{23} \rightarrow \infty .
\end{aligned}
$$

En terme de fonction de corrélation l'approximation de superposition implique donc que l'on peut écrire

$$
\begin{aligned}
g_{\alpha \beta \gamma}\left(\mathbf{r}_{1} \mathbf{r}_{2} \mathbf{r}_{3}\right) \simeq 1+h_{\alpha \beta}\left(r_{12}\right)+h_{\beta \gamma}\left(r_{23}\right) & + \\
& +h_{\gamma \alpha}\left(r_{31}\right) .
\end{aligned}
$$

Les termes négligés sont nécessairement des quantités petites par rapport à celles retenues. Ainsi que cela a été souligné par Meeron [8], l'approximation de Kirkwood écrite sous la forme (9) est une approximation ad hoc quand elle est utilisée dans des équations non linéarisées en $h_{\alpha \beta}\left(r_{12}\right)$.

2. Théorie de Debye Hückel et approximation sphérique moyenne (MSA). - Pour les systèmes qui nous intéressent le potentiel d'interaction $V_{\alpha \beta}\left(r_{12}\right)$ s'écrit

$$
V_{\alpha \beta}\left(r_{12}\right)=V_{\alpha \beta}^{*}\left(r_{12}\right)+V_{\alpha \beta}^{\mathrm{c}}\left(r_{12}\right)
$$

où $V_{\alpha \beta}^{*}\left(r_{12}\right)$ est un potentiel répulsif à courte distance tandis que $V_{\alpha \beta}^{\mathrm{c}}\left(r_{12}\right)$ désigne le potentiel coulombien $c_{\alpha} c_{\beta} / \varepsilon r_{12}$. Dans le cas des limites (11) on a, en portant (12) dans (4)

$$
k T \operatorname{grad}_{1} h_{\alpha \beta}\left(r_{12}\right)=-\operatorname{grad}_{1} V_{\alpha \beta}^{\mathrm{c}}\left(r_{12}\right)-\sum_{\gamma} n_{\gamma} \int \operatorname{grad}_{1} V_{\alpha \gamma}^{\mathrm{c}}\left(r_{13}\right) h_{\gamma \beta}\left(r_{32}\right) \mathrm{d} \mathbf{r}_{3} .
$$

Par intégration on peut écrire (14) sous la forme :

$$
h_{\alpha \beta}\left(r_{12}\right)=-\frac{V_{\alpha \beta}^{\mathrm{c}}\left(r_{12}\right)}{k T}+\sum_{\gamma} n_{\gamma} \int \frac{-V_{\alpha \gamma}^{\mathrm{c}}\left(r_{13}\right)}{k T} h_{\gamma \beta}\left(r_{32}\right) \mathrm{d} \mathbf{r}_{3} .
$$


Une telle relation a été utilisée dans [3]. La solution de (15) est la fonction de corrélation de Debye et Hückel :

$$
h_{\alpha \beta}^{\mathrm{DH}}=\frac{-c_{\alpha} c_{\beta}}{\varepsilon k T r} \exp -K_{\mathrm{D}} r
$$

La comparaison de (15) et (2) montre que la fonction de corrélation directe de Debye et Hückel $C_{\alpha \beta}^{\mathrm{DH}}$ est

$$
C_{\alpha \beta}^{\mathrm{DH}}=-\frac{V_{\alpha \beta}^{\mathrm{c}}}{k T} .
$$

Les fonctions (16) et (17) négligent totalement les contributions provenant des forces à courte distance. Dans le cas où $V_{\alpha \beta}^{*}\left(r_{12}\right)$ est un potentiel de type sphère dure, de diamètre $\sigma_{\alpha \beta}$, on sait que l'on a rigoureusement

$$
h_{\alpha \beta}\left(r_{12}\right)=-1 \text { si } r<\sigma_{\alpha \beta} .
$$

L'approximation sphérique moyenne [5] consiste à ajouter à ce résultat exact la forme approchée de la fonction de corrélation directe

$$
C_{\alpha \beta}^{\mathrm{MSA}}\left(r_{12}\right)=C_{\alpha \beta}^{\mathrm{DH}}\left(r_{12}\right) \text { pour } r>\sigma_{\alpha \beta} .
$$

L'avantage de l'approximation MSA est de fournir des expressions simples pour les grandeurs thermo- dynamiques que les ions soient de même dimension [5] ou non [9], [10]. Les fonctions de distribution radiale ont une expression analytique explicite lorsque les ions ont même diamètre [11]. Dans le modèle MSA le paramètre de Debye $K_{\mathrm{D}}$ est remplacé par :

$$
K^{\mathrm{MSA}}=\frac{\sqrt{1+2 K_{\mathrm{D}} \sigma}-1}{2 \sigma}=\frac{K_{\mathrm{D}}}{\sqrt{1+2 K_{\mathrm{D}} \sigma}+1}
$$

où $\sigma$ est le diamètre commun des ions. L'approximation MSA vérifie les conditions (7) et (8) mais se trouve en désaccord avec (6). Ceci entraîne, comme la théorie de Debye et Hückel que $h_{\alpha \alpha}\left(r_{12}\right)$ peut être fortement négatif pour les solutions très diluées. Différentes modifications ont été proposées pour corriger les imperfections de MSA [12], [13].

3. Les approximations de type HNC. - Alors que l'approximation MSA repose sur l'équation linéaire (14), nous allons montrer que l'on peut retrouver les approximations $\mathrm{HNC}$ à partir d'une équation non linéaire. Dans le cas des liquides purs un tel résultat a été obtenu par Abe [14], nous en donnons ici une démonstration plus simple et valable dans le cas des mélanges de particules. La démarche proposée n'est pas limitée au cas des particules chargées.

En portant (9) dans (4) on trouve

$$
\operatorname{grad}_{1}\left[k T \log g_{\alpha \beta}\left(r_{12}\right)+V_{\alpha \beta}\left(r_{12}\right)\right]+\sum_{\gamma} n_{\gamma} \int \operatorname{grad}_{1} V_{\alpha \gamma}\left(r_{13}\right) g_{\alpha \gamma}\left(r_{13}\right) h_{\gamma \beta}\left(r_{32}\right) \mathrm{d} \mathbf{r}_{3}
$$

Dans cette dernière relation on a substitué $h_{\gamma \beta}$ à $g_{\gamma \beta}$ car, à l'équilibre, la force totale moyenne s'exerçant sur chaque particule est nulle.

On peut établir une équation analogue pour $g_{\alpha \gamma}\left(r_{13}\right)$ et remplacer dans (21) le produit $\operatorname{grad}_{1} V_{\alpha \gamma}\left(r_{13}\right) g_{\alpha \gamma}\left(r_{13}\right)$. En poursuivant la substitution on trouve la relation :

$$
\begin{aligned}
\operatorname{grad}_{1}\left[k T \log g_{\alpha \beta}\left(r_{12}\right)\right. & \left.+V_{\alpha \beta}\left(r_{12}\right)\right]=\sum_{\gamma} n_{\gamma} \operatorname{grad}_{1} \int h_{\alpha \gamma}\left(r_{13}\right) h_{\gamma \beta}\left(r_{32}\right) \mathrm{d} \mathbf{r}_{3} \\
& -\sum_{\gamma} \sum_{\delta} n_{\gamma} n_{\delta} \int \operatorname{grad}_{1} h_{\alpha \delta}\left(r_{14}\right) h_{\delta \gamma}\left(r_{43}\right) g_{\gamma \alpha}\left(r_{31}\right) h_{\beta \gamma}\left(r_{23}\right) \mathrm{d} \mathbf{r}_{3} \mathrm{~d} \mathbf{r}_{4}+\cdots
\end{aligned}
$$

A l'aide de (3) on peut écrire

$$
\operatorname{grad}_{1}\left[k T \log g_{\alpha \beta}\left(r_{12}\right)+V_{\alpha \beta}\left(r_{12}\right)\right]=\operatorname{grad}_{1}\left[h_{\alpha \beta}\left(r_{12}\right)-C_{\alpha \beta}\left(r_{12}\right)\right]
$$

+ (termes faisant intervenir au moins deux fois la particule $\alpha$ ).

$\mathrm{Si}$ on se limite au premier terme du second membre, l'équation (23) se réduit à l'équation HNC. Pour les solutions électrolytiques, une telle expression a été résolue numériquement par Carley [15] et les résultats ont été discutés par Friedman et Rasaiah [16].

On a vu dans le paragraphe précédent que l'approximation de-superposition conduit pour $\boldsymbol{r}_{12}$ grand à la forme (16). Cependant cette relation ne vérifie pas la contrainte (6) et n'intègre pas la contribution de forces à courte distance. Il peut donc être intéressant de corriger les défauts de la théorie de Debye et Hückel en posant

$$
g_{\alpha \beta}\left(r_{12}\right)=\exp \left[-\beta V_{\alpha \beta}^{*}\left(r_{12}\right)+h_{\alpha \beta}^{\mathrm{DH}}\left(r_{12}\right)\right] .
$$

C'est la forme non linéarisée de la théorie de Debye et Hückel (Modèle DHX). On peut chercher une approximation meilleure en écrivant

$$
g_{\alpha \beta}\left(r_{12}\right)=\exp \left[-\beta V_{\alpha \beta}^{*}\left(r_{12}\right)+h_{\alpha \beta}^{\mathrm{DH}}\left(r_{12}\right)+\psi_{\alpha \beta}\left(r_{12}\right)\right]
$$


Dans (24) la fonction $\psi_{\alpha \beta}\left(r_{12}\right)$ peut être calculée en portant $g_{\alpha \beta}\left(r_{12}\right)$ ainsi définie dans (21). En tenant compte de ce que $h_{\alpha \beta}^{\mathrm{DH}}\left(r_{12}\right)$ vérifie l'équation (14) on a

$$
\begin{aligned}
k T \operatorname{grad}_{1} \psi_{\alpha \beta}\left(r_{12}\right) & +\sum_{\gamma} n_{\gamma} \int \operatorname{grad}_{1} V_{\alpha \gamma}\left(r_{13}\right) g_{\alpha \gamma}\left(r_{13}\right) h_{\gamma \beta}\left(r_{32}\right) \mathrm{d} \mathbf{r}_{3} \\
& -\sum_{\gamma} n_{\gamma} \int \operatorname{grad}_{1} V_{\alpha \gamma}^{\mathrm{c}}\left(r_{13}\right) h_{\gamma \beta}\left(r_{32}\right) \mathrm{d} \mathbf{r}_{3}=0 .
\end{aligned}
$$

Par une procédure de substitution analogue à celle qui nous a permis de passer de (22) à (23), on peut écrire (25) sous forme d'un développement. Si l'on néglige les termes faisant intervenir deux fois la particule $\alpha$ on aura un résultat identique à HNC mais que l'on pourra exprimer différemment. On a en effet immédiatement :

$$
\psi_{\alpha \beta}\left(r_{12}\right)=\sum_{\gamma} n_{\gamma} \int\left(h_{\alpha \gamma} h_{\gamma \beta}-h_{\alpha \gamma}^{\mathrm{DH}} h_{\gamma \beta}^{\mathrm{DH}}\right) \mathrm{d} \mathbf{r}_{3}-\sum_{\gamma} \sum_{\delta} n_{\gamma} n_{\delta} \int\left(h_{\alpha \delta} h_{\delta \gamma} h_{\gamma \beta}-h_{\alpha \delta}^{\mathrm{DH}} h_{\delta \gamma}^{\mathrm{DH}} h_{\gamma \beta}^{\mathrm{DH}}\right) \mathrm{d} \mathbf{r}_{3} \mathrm{~d} \mathbf{r}_{4}+\cdots
$$

Dans (26) les fonctions de corrélation se calculent à partir des fonctions de distribution radiale définies par l'équation (24). Le calcul de $\psi_{\alpha \beta}\left(r_{12}\right)$ à l'aide de (26) s'effectue en développant exp $\psi_{\alpha \beta}$ en série de $\psi_{\alpha \beta}$, cette fonction étant en elle-même écrite sous forme d'une série

$$
\psi_{\alpha \beta}\left(r_{12}\right)=\sum_{i=1}^{\infty} \psi_{\alpha \beta}^{(i)}\left(r_{12}\right)
$$

Les différents $\psi_{\alpha \beta}^{(i)}$ sont identifiés suivant les puissances de $n_{\alpha}$.

On a immédiatement

$$
\psi_{\alpha \beta}^{(1)}\left(r_{12}\right)=\sum_{\gamma} n_{\gamma} \int\left[\exp \left[-\beta V_{\alpha \gamma}^{*}+h_{\alpha \gamma}^{\mathrm{DH}}\right] \cdot \exp \left[-\beta V_{\gamma \beta}^{*}+h_{\gamma \beta}^{\mathrm{DH}}\right]-h_{\alpha \gamma}^{\mathrm{DH}} h_{\gamma \beta}^{\mathrm{DH}}\right] \mathrm{d} \mathbf{r}_{3} .
$$

Si on limite $\psi_{\alpha \beta}$ à $\psi_{\alpha \beta}^{(1)}$ alors la fonction de distribution radiale (24) se réduit à une approximation discutée dans [16], [17]. Par une approche quelque peu différente Falkenhagen et Ebeling [3] ont proposé un modèle conduisant à

$$
g_{\alpha \beta}\left(r_{12}\right)=\exp \left[-\beta V_{\alpha \beta}^{*}+h_{\alpha \beta}^{\mathrm{DH}}\right] \cdot\left(1+\psi_{\alpha \beta}^{(1)}\left(r_{12}\right)\right) .
$$

Les approximations qui conduisent à (26) étant strictement identiques à celles qui sont utilisées pour déduire l'équation HNC, les équations (24) et (26) constituent une autre forme de l'équation HNC. Si l'on introduit la fonction

$$
\varphi_{\alpha \beta}\left(r_{12}\right)=\exp \left[-\beta V_{\alpha \beta}^{*}+h_{\alpha \beta}^{\mathrm{DH}}\right]-1-h_{\alpha \beta}^{\mathrm{DH}}
$$

on peut montrer que (26) est identique à la version proposée par Alnatt [18] pour l'équation HNC des milieux ioniques.

4. Conclusions. - Les équations HNC et MSA sont d'une grande importance dans l'étude des solutions ioniques [2]. Ces deux équations peuvent être déduites simplement de l'approximation de superposition de Kirkwood. Elles reposent toutes les deux sur une extrapolation à toute distance de résultats valables à longue distance. Cependant ces extrapolations sont différentes puisque dans le cas de $\mathrm{HNC} g_{\alpha \beta}\left(r_{12}\right)$ obéit à une équation non linéaire en terme des différentes fonctions de corrélation alors que MSA résulte d'une équation linéarisée.

Si l'approximation de superposition suffit pour retrouver MSA, elle ne suffit pas pour aboutir à HNC.

Il semble difficile a priori de prévoir le domaine de validité de chacune de ces deux approches. Toutefois le fait que MSA ne permette pas de retrouver la forme exponentielle de $g_{\alpha \beta}\left(r_{12}\right)$ quand la concentration tend vers zéro (non respect de la condition (6)) montre que cette approche sera très peu satisfaisante pour décrire le comportement de solutions relativement diluées. Une comparaison des prédictions de HNC et MSA d'une part et de résultats de Monte Carlo d'autre part a été effectuée dans plusieurs travaux récents [2], [11], [19], [21].

Les techniques de développement ou diagrammes ont conduit à des approches autres que HNC ou MSA c'est par exemple ORPA, MEX ou EXP [2]. Ces techniques constituent un outil très puissant pour l'étude des propriétés structurales des liquides ioniques. Cependant si l'on considère les phénomènes de transport, les résultats obtenus par l'intermédiaire des diagrammes sont de portée très limitée. Les mises en équation que nous avons présentées ici et qui reposent sur des solutions approchées d'équations intégrales pourront peut être aider à élaborer de nouvelles approximations pour le calcul des phénomènes de transport. 


\section{Bibliographie}

[1] DebYe, P., HÜCKel, E., Phys. Z. 24 (1923) 185.

[2] Rasaiah, J. C., J. Solution Chem. 2 (1973) 301.

[3] Falkenhagen, H., Ebeling, W., Ionic Interactions I (Academic Press), ed.S. Petrucci.

[4] RICE, S. A., GRAY, P., The statistical mechanics of simple liquids (Interscience publishers) 1965.

[5] Waizman, E., Lebowitz, J. L., J. Chem. Phys. 56 (1972) 3086, 3093.

[6] ResiboIs, P. M. V., Electrolyte theory (Harper and Raw) 1968.

[7] Stillinger, F. H., Lovett, R., J. Chem. Phys. 48 (1968) 3858.

[8] Meeron, E., Electrolytes (Pergamon Press) 1962.

[9] Grigera, J. R., Blum, L., Chem. Phys. Lett. 38 (1976) 486.

[10] Triolo, R., Grigera, J. R., Blum, L., J. Phys. Chem. 80 (1976) 1858.
[11] Badial, J. P., Lestrade, J. C., Rosingerg, M. L., Chem. Phys. Lett. 34 (1975) 156.

[12] HøYe, J. S., Lebowitz, J. L., Stell, G., J. Chem. Phys. 61 (1974) 3253.

[13] Stell, G., Sun, S. F., J. Chem. Phys. 63 (1975) 5333.

[14] ABE, R., Prog. Theor. Phys. (Kyoto) 19 (1958) 407.

[15] CaRley, D. D., J. Chem. Phys. 46 (1967) 3783.

[16] Rasaiah, J, C., Friedman, H., J. Chem. Phys. 48 (1968) 2742.

[17] Rasaiah, J. C., J. Chem. Phys. 56 (1972) 3071.

[18] Allnat, A. R., Mol. Phys. 8 (1964) 533.

[19] Rasaiah, J. C., Card, D. N., Vallbau, J. P., J. Chem. Phys. 56 (1972) 248.

[20] Andersen, H. C., Chandler, D., Weeks, J. D., J. Chem. Phys. 57 (1972) 2626.

[21] Larsen, B., J. Chem. Phys. 65 (1976) 3431. 